\title{
Dağıtım sistemleri için çok fonksiyonlu statik senkron kompanzatör
}

\author{
Lütfü Sarıbulut $^{1 *}$, Ahmet Teke ${ }^{2}$, Mohammad Barghı Latran ${ }^{2}$ \\ ${ }^{1}$ Adana Bilim ve Teknoloji Üniversitesi, Elektrik-Elektronik Mühendisliği Bölümü, Seyhan, Adana, 01180, Türkiye \\ ${ }^{2}$ Çukurova Üniversitesi, Elektrik-Elektronik Mühendisliği Bölümü, Sarıçam, Adana, 01330, Türkiye
}

Ö N E Ç I K A N L A R

- Güç kalitesi problemlerinin kompanzasyonu için D-STATKOM

- D-STATKOM için Genel Dalgacık Dönüşüm tabanlı kontrolcü tasarımı

- DA kondansatörünün gerilim kontrolü için Bulanık Mantık tabanlı kontrolcü tasarımı

Makale Bilgileri

Geliș: 23.08.2015

Kabul: 04.03.2016

DOI:

10.17341/gummfd.11948

Anahtar Kelimeler:

Dağıtım sistemi statik senkron kompanzatör, dalgacık dönüşümü,

harmonik,

reaktif güç kompanzasyonu

\section{ÖZET}

Güç kalitesi problemleri, elektronik cihazların ve hassas yüklerin zarar görmesine ve verimsiz çalışmalarına neden olmakta ve önemli ölçüde ekonomik kayıplara yol açmaktadır. Bu problemlerin giderilmesi için, sistem dinamiklerindeki değişimlere hızlı cevap vermesi ve güç sistemi parametrelerini kontrol edebilmesi özelliklerinden dolayı Dağıtım Sistemi Statik Senkron Kompanzatör (D-STATKOM) yaygın olarak kullanılmaktadır. Bu araştırma çalışmasında, Genel Dalgacık Dönüşümüne dayalı bir kontrol yöntemine sahip D-STATKOM ilk defa önerilmiş olup, dağıtım sistemlerindeki en önemli güç kalitesi problemlerinden olan dengeli/dengesiz gerilim dalgalanmaları, akım/gerilim harmonikleri ve reaktif güç eş zamanlı olarak kompanze edilmiştir. Bu çalışma ile birlikte genel dalgacık algoritması ilk defa matematiksel olarak formüle edilmiştir. Çeşitli durum çalışmalarıyla önerilen yöntemin performansı PSCAD/EMTDC programı kullanılarak test edilmiştir.

\section{Multi-functional static synchronous compensator for distribution systems}

\section{H I G H L I G H T S}

- $\quad$ Design of D-STATCOM for compensation of power quality problems

- General Wavelet Transform based controller design for D-STATCOM

- $\quad$ Fuzzy Logic based controller design for voltage control of DC capacitor

Article Info

Received: 23.08.2015

Accepted: 04.03.2016

DOI

10.17341/gummfd.11948

Keywords:

Distribution static synchronous compensator wavelet transform, harmonics, reactive power compensation

\section{GIRIŞ (INTRODUCTION)}

Dünyada ve ülkemizde, elektrik enerjisine olan talebin hızla artması ve gelişen teknoloji ile birlikte elektrik güç sistemlerine bağlı yük karakteristiklerindeki değişimler, elektrikte güç kalitesi (GK) konusunun önem kazanmasına neden olmuştur. GK problemleri, elektrik enerjisi kullanan cihazların hatalı çalışmasına, son kullanıcıların ve yüklerin zarar görmesine sebep olan gerilim veya akımın genlik, frekans ve dalga formundaki değişimler olarak tanımlanmaktadır. Elektrik dağıtım sistemlerinde en sık karşılaşılan elektrik güç kalitesi problemleri gerilim çukuru,

\footnotetext{
* Sorumlu Yazar/Corresponding author: 1saribulut@adanabtu.edu.tr / 03224502030
} 
gerilim tepeleri, gerilim ve akım harmonikleri, düşük güç faktörü, gerilim ve akım dengesizlikleridir. $\mathrm{Bu}$ GK problemlerinin etkili bir şekilde telafi edilmesi, iletim ve dağıtım sistemlerinin düşük maliyetlerle işletilmesine ve tüketicilere daha kaliteli bir elektrik enerjisinin sunulmasına imkân sağlamaktadır. GK problemlerinin düzeltilmesinde hızlı tepki veremeyen geleneksel kompanzasyon cihazları yetersiz kaldığından, güç elektroniği tabanlı kompanzasyon cihazlarının geliştirilmesine gereksinim duyulmuştur. Son yıllarda akım ve gerilimdeki kalite problemlerinin düzeltilmesi için şebekeye seri/paralel bağlantılı cihazlar geliştirilmiştir. Bunlara örnek olarak harmoniklerin elenmesi için Aktif Güç Filtresi (AGF), reaktif güç akışının kontrolü ve ortak bağlantı noktadaki gerilimin düzenlenmesi için D-STATKOM verilebilir. Elektrik güç sistemlerinde karşılaşılan farklı güç kalitesi problemlerinin artması, seri/paralel bağlantılı cihazların maliyetlerinin yüksek olması ve alan kapmalarından dolayı en az güç elektroniği elemanıyla (örnek olarak tek evirgeç) birçok GK probleminin giderilmesi ihtiyacı, son yıllarda D-STATKOM sistemlerinin çok-fonksiyonlu olarak tasarlanmasını hızlandırmıștır. Bu çalıșmaların sonucunda akım ve gerilim harmonikleri/dengesizlikleri, geçici rejim olaylarının sönümlenmesi ve reaktif kompanzasyonu gibi birçok GK problemini düzeltebilecek şekilde geliştirilmiştir. Literatürde, son yıllarda D-STATKOM ile ilgili çalışmaların sayısı artmış olup, kontrolcüsü zaman veya frekans düzleminde referans sinyalleri üretilerek kompanzasyonu gerçekleştirmektedir. Örnek olarak ani gerilim ve/veya akım vektörlerinin zamana bağlı sinyalleri algilanır ve bu sinyaller DQ Dönüşümü [1-4], PQ Dönüşümü [5-7], geri yayılım [8], karma gözlemci tabanlı [9], vektör kuantalamalı öğrenme [10], uyarlamalı eşzamanlı referans çıkarma [11-12], yapay sinir ağları tabanlı Anti-Hebbian algoritması [13], üçgen fonksiyon karakteri [14], ayarlanabilir adımlı en küçük kareler [15], Adaline yapay sinir ağları [16] ve Kalman filtre [17] gibi yöntemler kullanılarak bileşenlerine ayrıştııılır. Yukarıda verilen yöntemler kullanılarak elde edilen değerler, kompanzasyon sinyallerini üretmek için Oransal-İntegral (OI) veya Oransal-İntegral-Türevsel (OIT) gibi çeşitli kontrolcüler kullanılarak işlenir. Evirgeçteki anahtarların tetiklenme süreleri akım kontrol teknikleri, doğrusal ve/veya doğrusal olmayan kontrol stratejileri ile hesaplanmaktadır. Doğrusal kontrol yöntemlerinde OI denetleyici, rampa karşılaştırmalı akım kontrol, eşzamanlı vektör OI kontrol, durum geri beslemeli kontrol [18], kestirimci kontrol ve sonlu-zaman (deadbeat) [19] gibi kontrol yöntemleri kullanılmaktadır. Doğrusal olmayan denetleyicilerde ise histerezis kontrol [20-23], yapay sinir ağları [24], bulanık mantık [25-26], admittans tabanlı algoritmalar [27], serbest-model temelli kestirimci [28], kayan kip [20-30], melez genetik algoritma tabanlı [31], gelişmiş lineer sinüs izleyici tabanlı [32], yapay bağışıklık sistemi [33], delta modülasyon (DM), darbe DM akım kontrol ve çevrimiçi optimize edilmiş gibi yöntemler kullanılmıştır [34]. Bu çalışmada, Dalgacık Dönüşümü (DD) tabanlı kontrol yöntemine sahip bir DSTATKOM geliştirilmiş olup, dağıtım sistemlerinde sıklıkla görülen dengeli/dengesiz gerilim dalgalanmaları, akım/gerilim harmonikleri ve reaktif güç kompanze edilmiştir. D-STATKOM'un evirgecinde bulunan doğru akım (DA) kondansatörün gerilim kontrolü, Bulanık Mantık tabanlı kontrolcü ile sağlanmıştır. Bu çalışma ile tek bir evirgeç kullanılarak en az maliyetle (tek evirgeç kullanarak) birçok GK probleminin eş zamanlı olarak giderilmesi sağlanmıştır.

\section{STATKOM'UN GÜÇ DEVRESI (THE POWER CIRCUIT OF D-STATCOM)}

Benzetim çalışmalarında gerçekleştirilen D-STATKOM 6darbeli evirgeç (inverter) yapısında ve 3 -faz $5 \mathrm{kVA}$ gücünde tasarlanmış olup, $380 \mathrm{~V}_{\text {hat-hat }}-4 \mathrm{kVA}$ yüke sahip bir dağıtım hattına bağlanmıştır. Dağıtım hattı " $1 \Omega+10 \mathrm{mH}$ " yükü ile temsil edilmiş olup, [1]'de verilen parametreler kullanılmıştır. Devrede harmonik kaynağı olarak

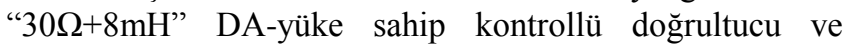
dengesiz faz-akımları oluşturulması için dengesiz yük modeli kullanılmıştır. Evirgeç çıkışına darbe-genişlik modülasyonunda oluşan harmonikleri azaltmak için " $35 \Omega+10 \mathrm{mH}+35 \mu \mathrm{F}$ " değerlere sahip L-C-R filtresi tasarlanmıştır. $\mathrm{Bu}$ değerler [35]'de verilen hesaplama yöntemleri ile belirlenmiştir. Devreye belirli aralıklarla kesici-1 (BRK1) ile dengesiz yük; kesici-2 (BRK2) ile DSTATKOM modülleri devreye alınıp-çıkarılarak durum çalışmalarında sistem dinamikleri incelenmiştir. Benzetimi yapılan güç sisteminin detaylı gösterimi Şekil 1'de sunulmuştur.

\section{D-STATKOM İÇIN GELIŞTIIRILLEN KONTROL METODU (THE PROPOSED CONTROL METHOD OF D-STATCOM)}

D-STATKOM'un kontrolünde ilk olarak toplam yük akımlarının $\left(i r_{a}, i r_{b}, i r_{c}\right)$ pozitif bileşenleri $\left(i r_{a-p}, i r_{b_{-} p}, i r_{c_{-} p}\right)$ bulunur [36] ve daha sonra her bileşen önerilen DD yöntemine tabi tutularak temel harmonikleri çıarılır. Dalgacık sürecinde, giriş sinyalinin her periyodunda 200 örnek alınmakta olup, 2 kat ayrıştırma ve tekrar oluşturma sürecine tabi tutulmaktadır. Daubechies, Meyer ve Haar gibi farklı dalgacık filtreleri denenmiş ve en iyi performans Haar filtresinden elde edilmiştir. Elde edilen çıkış sinyali, giriş sinyali ile kalibre edilmesi için katsayı $\left(\mathrm{k}_{\mathrm{wvlt}}=1,3538\right)$ ile çarpılmaktadır. Önerilen DD yöntemi ile ilgili detaylı bilgiler bir sonraki bölümde sunulmuștur. D-STATKOM'un evirgecinde bulunan kapasite, aktif güç kullanılarak DA gerilimini $\left(v_{d c l}\right)$ sabit tutabildiğinden, dalgacık sürecinden elde edilen pozitif-birleşenlerin temel harmoniklerine $\left(i r_{a_{-} p_{-} r}, i r_{b_{-} p_{-}}, i r_{c_{-} p_{-} r}\right)$ Park Dönüşüm (D-Q) uygulanarak ' $\mathrm{D}$ ' birleşenleri elde edilir [37] ve kapasitör gerilim kontrol modülünden elde edilen sinyalden $\left(v_{d c l \text { err }}\right)$ çıkarılır. Böylece kapasitör gerilimi için gerekli olan aktif güç kontrol yöntemine dâhil edilmiş olur. DQ uygulamasında giriş sinyali Clarke $(\alpha-\beta)$ Dönüşümü 'nün ' $\alpha$ ' birleșeni olarak kabul edilmiş ve ' $\alpha$ ' birleşeni $90^{\circ}$ geciktirilerek ' $\beta$ ' birleşeni elde edilmiştir. ' $\mathrm{D}$ ' birleşeni harmonik etkilerden korumak 


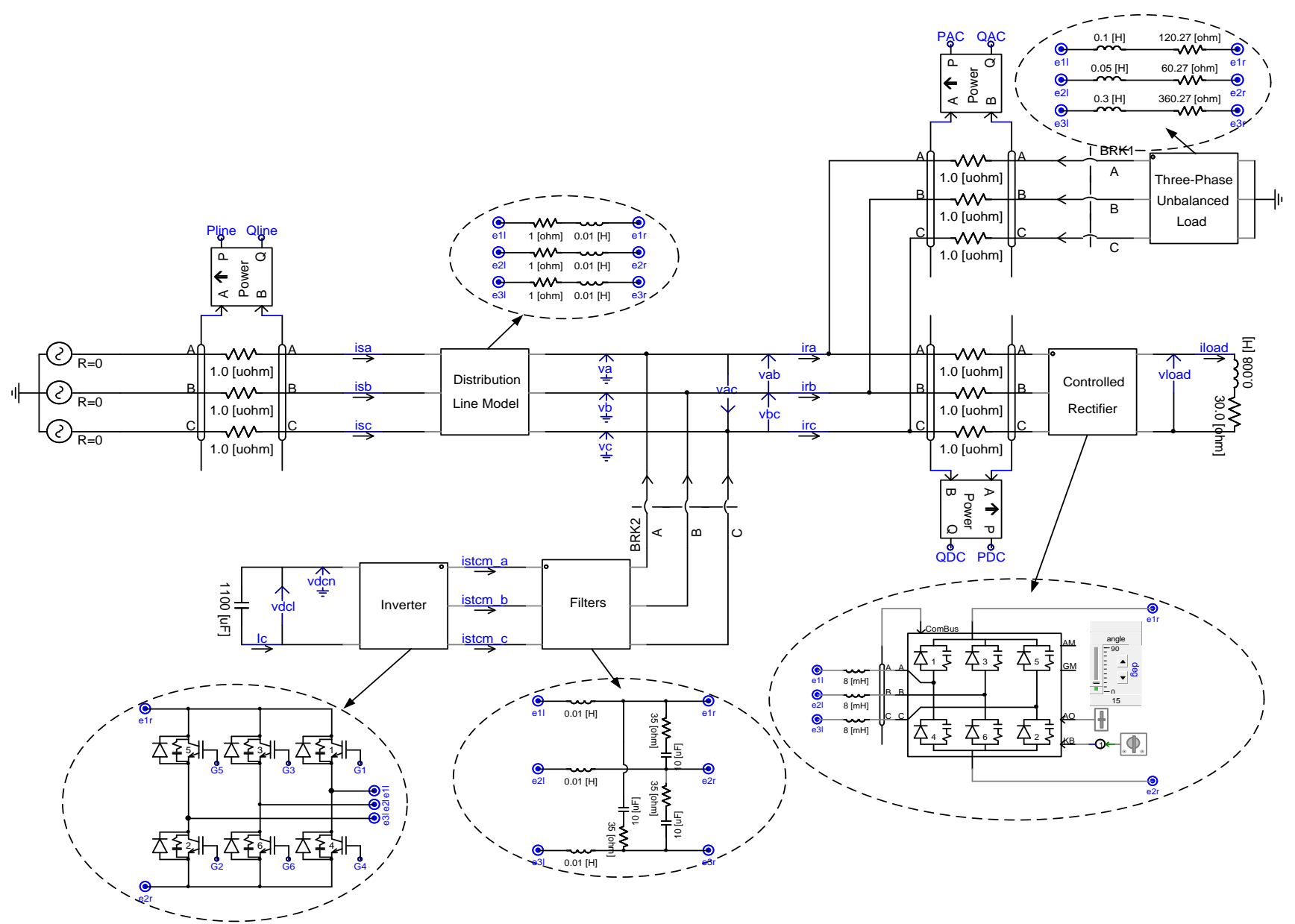

Şekil 1. Test sistemi ve geliştirilen D-STATKOM güç devresi (Test system and the power circuit of the proposed D-STATCOM)

için kesim frekans $10 \mathrm{~Hz}$ ayarlanmış alçak-geçiren filtreden geçirilmiştir. Park dönüşümünde, sistem geriliminin faz açısı $(\varphi)$ kullanılmış olup, bunun için PSCAD/EMTDC kütüphanesinde bulunan Faz-Kilitlemeli Döngü (PLL) modeli kullanılmıştır [38]. Kapasitör gerilim kontrolü, anlık olarak ölçümü yapılan $v_{d c l}$ geriliminin $v_{d c l_{-} r e f}$ den $(0,650 \mathrm{kV})$ çıkarılmasıyla elde edilen fark değerinin, daha sonra Bulanık Mantık Kontrol (BMK) yönteminden geçirilmesiyle elde edilen sinyal ile gerçekleştirilmektedir. Kullanılan BMK yöntemi [39] makalesindeki yöntem olup, DSTATKOM'un kontrol sistemine dâhil edilmiş ve çıkışını $\mathrm{k}_{\mathrm{dc}}$ katsayısı $\left(\mathrm{k}_{\mathrm{dd}}=0,235\right)$ ile çarparak $\mathrm{DQ}$ sürecine dâhil edilmiştir.

Ters DQ dönüșümünden elde edilen ' $\alpha$ ' bileșeni harmonik etkilerden korumak için kesim frekansı $50 \mathrm{~Hz}$ ayarlanmış alçak-geçiren filtreden geçirilir. Elde edilen sinyal a-fazı hata akımı $\left(i r_{a_{-} e r r}\right)$ olarak kabul edilir ve D-STATKOM'un a-fazı akımı $\left(i_{\text {stscm a }}\right)$ ile toplanır. Toplam sonucu $i r_{a}$,'dan çıkarılarak a-fazı için kompanzasyon sinyali $\left(i_{\text {cmp_a }}\right)$ elde edilir. Daha sonra kompanzasyon sinyali $2,5 \mathrm{kHZ}$ frekansa sahip taşıyıcı sinyali ile karşılaştırılarak IGBT'ler için kontrol sinyalleri üretilir. Tasarlanan kontrol algoritması detaylı olarak Şekil 2'de sunulmuştur.

\section{4. ÖNERİLEN DALGACIK DÖNÜŞÜMÜ YÖNTEMİ (THE PROPOSED WAVELET TRANSFORM METHOD)}

Güç sistemlerinde zamanla frekansı değișen sinyallerin belirlenmesinde ve karakterize edilmesinde DD kullanımı artmaktadır. DD, durağan veya durağan olmayan sinyaller için farklı ölçeklerdeki çözünürlük seviyelerinde tek bir fonksiyonu genişleterek ayrıştıran bir yöntemdir. Özellikle, sinyal birleşenlerinden düşük frekanslı olanlar büyük zaman aralıklarında, yüksek frekanslı olanlar küçük zaman aralıklarında ayrıştırılırlar. Bir başka deyişle, sinyalin ölçeklenebilir bir zaman-frekans gösterimi ile analizini gerçekleştirir ve diğer yöntemler tarafından belirlenemeyen frekans birleşenlerinin ölçeklenebilir filtre pencereleri ile ortaya çıarır. Ayrıca, analiz için birkaç frekans bandının kullanılması gerekliyse, tüm spektrumu hesaplamaya gerek bulunmamaktadır ve düşük frekans spektrum kısımlarında oldukça hızlı olmaktadır [40]. Giriş sinyali alçak-yüksek geçirgen filtrelerle frekans sinyallerine ayrılarak istenilen frekans aralığına ulaşılır ve gerekli harmonik birleşen elde edilir. $x(t)$ giriş sinyali ve $x(n)$ dizisi, $x(t)$ sinyalinin bir periyodundan elde edilen örnek dizisi olsun. O zaman $x(n)$ Eş. (1) ile ifade edilebilir. Burada $s n$ örnekleme sayısını ifade etmektedir. 


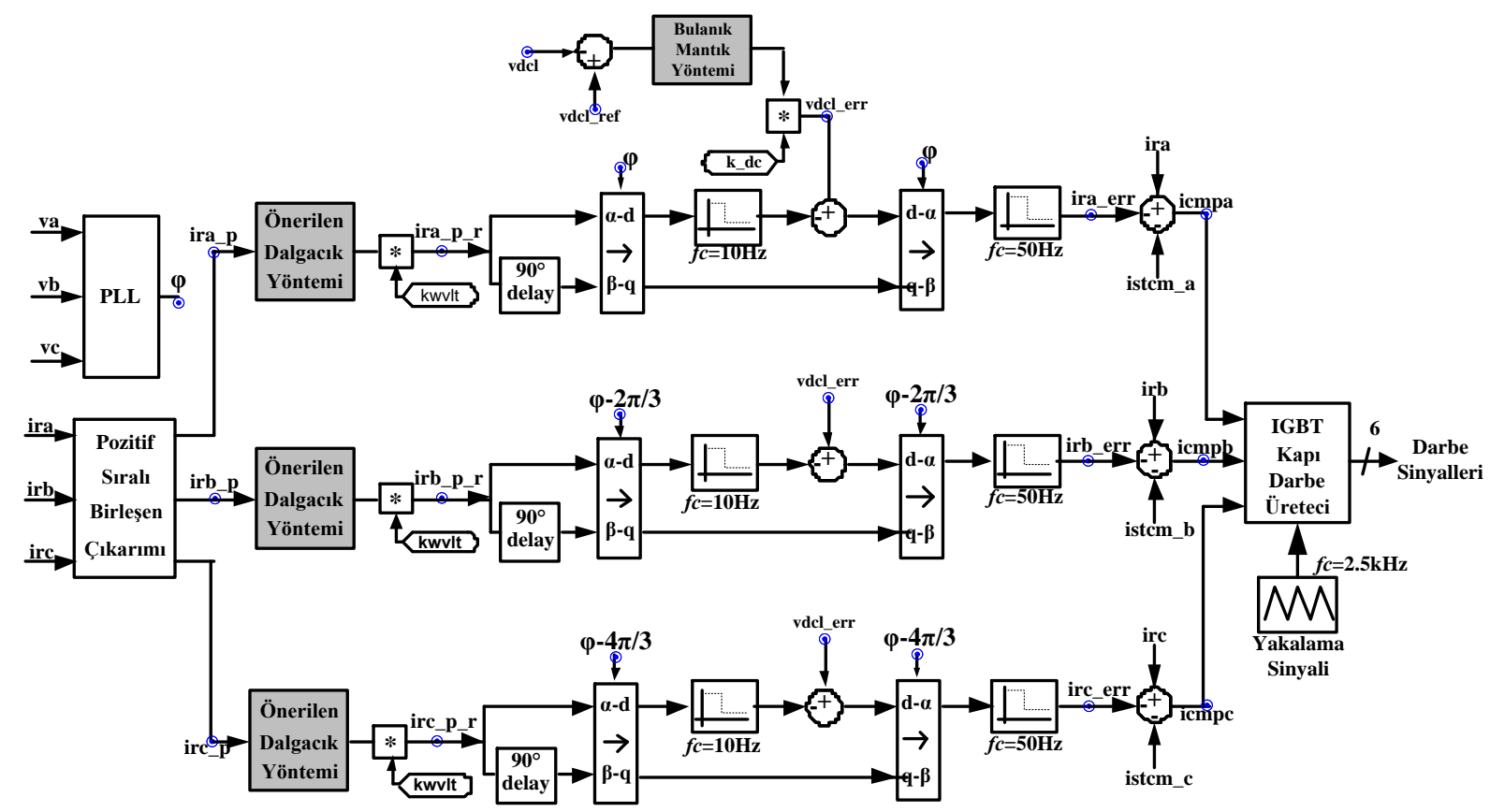

Şekil 2. D-STATKOM'un kontrol algoritması (Control algorithm of D-STATCOM)

$$
x(n)=\sum_{n=1}^{s n} x(n T)
$$

Her bir örnekleme anında dizinin tüm terimleri kendisinden önceki bir alt endekse sahip dizi terimine aktarılır, alınan yeni örnek en büyük endeksli dizi teriminde saklanarak $x(n)$ güncellenir. Güncelleme işlemden hemen sonra Ayrıştırma Süreci'nde (AS) kullanılacak olan $x_{d l}(n)$ dizisi Eş. (2) kullanılarak hazırlanır. Burada $d l$ AS'nin seviyesi, $d_{2}$ kullanılan filtre derecesinin $\left(d_{1}\right)$ yarıs1, $s(d l) \quad d l$ seviyesindeki $s n$ değeridir.

$$
x_{d l}(n)=\sum_{n=d_{2}}^{1} x(n)+\sum_{n=1}^{s(d l)} x\left(n+d_{2}\right)+\sum_{n=s(d l)}^{s(d l)-d_{2}} x\left(n+d_{2}+s(d l)\right)
$$

Eş. (2)'de kullanılan $s(d l)$ 'in değeri, Eş. (3)'te kullanılarak hesaplanır. Burada $d l$ değeri sıfırdan başlar.

$$
s(d l)=s n / 2^{d l}
$$

Eş. (3)'e göre, AS'de $d l$ arttıkça $s n$ değeri azalır. Başka bir deyişle, yüksek endekse sahip Ayrıştırma Seyisi (ASY) bir alt endekse göre $s n$ değerinin yarısına sahiptir. Her bir ASY'de $x_{d l}(n)$, alçak-geçiren $(l w(k))$ Eş. (4) ve yüksekgeçiren $(h(k))$ Eş. (5) filtrelerden geçirilerek $x(n)$ frekans spektrumda ikiye ayrılmış olur. Alçak frekans filtre çıkışlarına $x_{d l}(n)$ serisinin Yaklaşımları (YA) olarak isimlendirilir ve frekans spektrumunun orta frekansından düşük frekansları içerir. İkinci kısım ise yüksek filtre çıkışları olup $x_{d l}(n)$ serisinin Detayları olarak (DE) olarak isimlendirilir ve frekans spektrumunun orta frekansından yüksek frekansları içerir. AY'nin YA ve DE katsayıları Eş. (4) ve Eş. (5) kullanılarak elde edilir. Burada $l w(k)$ ve $h(k)$ sırasıyla alçak ve yüksek geçiren filtrelerin katsayılarıdır.

$$
\begin{array}{r}
x_{d l-l w}(n)=\sum_{n=1}^{s(d l)+d_{1} / 2} \sum_{k=1}^{d_{1}} x_{d l}(k+2 \cdot n) \cdot l w(k) \\
s(d l)+d_{1} / 2 \\
x_{d l-h}(n)=\sum_{n=1}^{d_{1}} x_{k=1}(k+2 \cdot n) \cdot h(k)
\end{array}
$$

Gerekli olan frekans birleşenine $\left(f_{\text {req }}\right)$ ulaşılana kadar $d l$ 'nin değeri arttırılır ve dizisi sayısı $d l$ ile orantılı olarak azalır. Her bir ASY'de Eş. (2) + Eş. (4) veya Eş. (2) + Eş. (5) ile en yüksek $d l$ değerine ulaşana kadar tekrarlanır. Bu sürecin sonunda elde edilen YA ve DE katsayılarından, $f_{\text {req }}$ sahip olan birleşeni barındıran bir tanesi Yeniden Yapılandırma sürecine (YY) tabi tutulur. Yeniden yapılandırma derecesinin değeri $(r l), d l$ en son değerinden başlayarak sıfira kadar azaltılırken, dizinin $s n$ değeri her $r l$ 'nin değişiminde bir önceki seviyenin 2 katına çıkarılır. $x_{d l-l w}(n)$, AS'nin sonunda elde edilen dizilerden olsun ve $f_{\text {req }}$ sahip sinyali veren yaklaşım katsayılarını versin. YY 'için Eş. (6) kullanılarak $x_{d l-l w}(n)$ 'den yeni bir dizi $\left(x_{r l}(n)\right)$ oluşturulur. Burada $x_{d l-l w}(n)$ dizisinin terimleri $x_{r l}(n)$ dizisinin tekendeksli terimlerine atanırken, çift indeksli terimler sıfir yapilir.

$x_{r l}(2 . n+1)=\left.x_{d l-l w}(n)\right|_{n=1} ^{s(d l)}$ 
Daha sonra, $x_{r l}(n)$ aynı dalgacık filtrelerinden geçirilerek YY süreci başlamış olur. Bu sürecin YA ve DE katsayıları Eş. (7) ve Eş. (8) kullanılarak elde edilir. Burada $d l$ 'nin değeri en yüksek değerinden başlayarak sıfıra kadar azalır.

$$
\begin{aligned}
& x_{r l_{-} l w}(n)=\sum_{n=1}^{s(d l)+d_{1}} \sum_{k=1}^{d_{1}} x_{r l}(k+n) \cdot l w(k) \\
& x_{r l_{-} h}(n)=\sum_{n=1}^{s(d l)+d_{1}} \sum_{k=1}^{d_{1}} x_{r l}(k+n) \cdot h(k)
\end{aligned}
$$

Her bir Yeniden Yapılandırma Seviyesinde (YYS) Eş. (6) + Eş. (7) veya Eş. (6) + Eş. (8) eşitlikleri, $d l$ değeri sıfır olana kadar tekrarlanır. Bu sürecin sonunda, sn elemana sahip $x_{r l}(n)$ ve $f_{r e q}$ frekansına sahip birleşen $\left(x_{r l}(l)\right)$ elde edilmiş olunur. DD'de örnekleme frekansı $\left(f_{\text {smp }}\right)$ ve $f_{\text {req }}$, $d l$ ve $r l$ sevilerinin belirlenmesinde önemli rol oynamaktadır. Örneğin $10 \mathrm{kHz}$ ile örneklenmiş tek-harmonikleri içeren bir sinüs-sinyalimiz olsun. $50 \mathrm{~Hz}$ frekansa sahip temel birleşenini 7 adet ayrıştırma ve yeniden yapılandırma seviyelerinin gerçekleştirilmesinden sonra elde edilebilmektedir. Ayrıştırma ve yeniden-yapılandırma süreçlerinin sevilerinin artmasıyla DD algoritmasını daha karmaşık hale getirmekte ve mikro-işlemciler için hesaplama yükünü arttırmaktadır. $\mathrm{Bu}$ durum ise kontrolcülerin tepki süresini geciktirmektedir. $\mathrm{Bu}$ çalışmada, temel harmoniği çıkartmak için matematiksel hesaplama yükü azaltılarak daha hızlı tepki süresine sahip geleneksel DD tabanlı yeni bir yöntem önerilmiştir. Bu yöntem, çıkış sinyalini geri-bildirim bilgisi olarak kullanan OI kontrol sistemi göz önüne alınarak geliştirilmiştir. Önerilen yöntem bu bilgiyi, YYS'nin son seviyesinde Eş. (7)'nin çıkışında elde edilen katsayıları Eş. (1)'de giriş olarak kullanılmasıyla gerçekleştirilmektedir. Bu işlem Eş. (1)'in hemen ardına Eş. (9) kullanarak gerçekleştirilir. Burada $x_{\text {freq }}(n)$ önerilen yöntemin çıkışında elde edilen, $f_{\text {req }}$ sahip birleşen katsayıları, $y(s n)-x(s n)$ değişkenleri $y(n)$ ve $x(n)$ dizilerinin en sonuncu terimlerini ifade eder.

$$
\left.y(n)\right|_{n=1} ^{s n-1}+y(s n)=\left.x_{f r e q}(n)\right|_{n=1} ^{s n-1}+x(s n)
$$

Önerilen yöntemin her örnekleme zamanında $x(\mathrm{~s} n)^{\prime}$ de tutulan yeni terimi $y(\mathrm{~s} n)$ 'ye, YYS'nin son seviyesinde elde edilen 'sn-1'adet $x_{\text {freq }}(n)$ 'nin katsay1s1 aynı sayıda $y(n)$ 'nin terimine atanır ve daha sonra sırasıyla ayrıştırma ve yeniden-yapılandırma süreçlerine tabi tutulur. Böylelikle sistem çıkışını girişi ile etkileştirerek PI kontrol sistemlerindeki gibi geri-bildirim sağlanmış olunur. Bu metotla $10 \mathrm{kHz}$ ile örneklenmiş bir periyodik sinyalin $50 \mathrm{~Hz}$ 'lik birleşeni 2 adet ayrıştırma ve yenidenyapılandırma süreçlerin gerçekleştirilmesinden sonra elde edilebilmektedir. Seviye sayılarının azaltılması sonucu, DD algoritması ilk haline göre daha hızlı tepki vermekte ve mikro-işlemciler için hesaplama yükünü çok daha azaltılmaktadır. Kullanılan PSCAD/EMTDC programında kullanıcılar tarafinda oluşturulan modellere, Fortran kodları kolaylıkla dâhil edilebilmektedir. Bu özellik kullanılarak önerilen yöntem, Fortran kodları ile kullanıcı ara yüzü oluşturulmuş ve programdan alınan görüntü ile görsel olarak Şekil 3 'te sunulmuş olup okuyucular tarafindan daha kolaylıkla anlaşılabilmesi için yukarıda verilen eşitliklerin kullanım yerleri ayrıştırma ve yeniden yapılandırma süreçleri göz önünde bulundurarak belirtilmiştir.

\section{5. ÖNERILEN SISTEMIN BENZETIMM TESTLERİ (THE SIMULATION TESTS OF THE PROPOSED SYSTEM)}

Yapılan durum çalışmalarında doğrultucu ve DA yükler sabit olarak devrede olup, dengesiz yükler devreye bir ayırıcı ile eklenip veya çıkarılabilmektedir. Ayrıca DSTATKOM'un dengesiz sistem gerilimlerindeki performansını incelenmesi amacıyla sisteme bağlı gerilim kaynaklarının değerleri son durum çalışmasında değiştirilmiştir. Sistemin D-STATKOM devre dış1 durumundaki güç değerleri Tablo 1'de sunulmuştur.

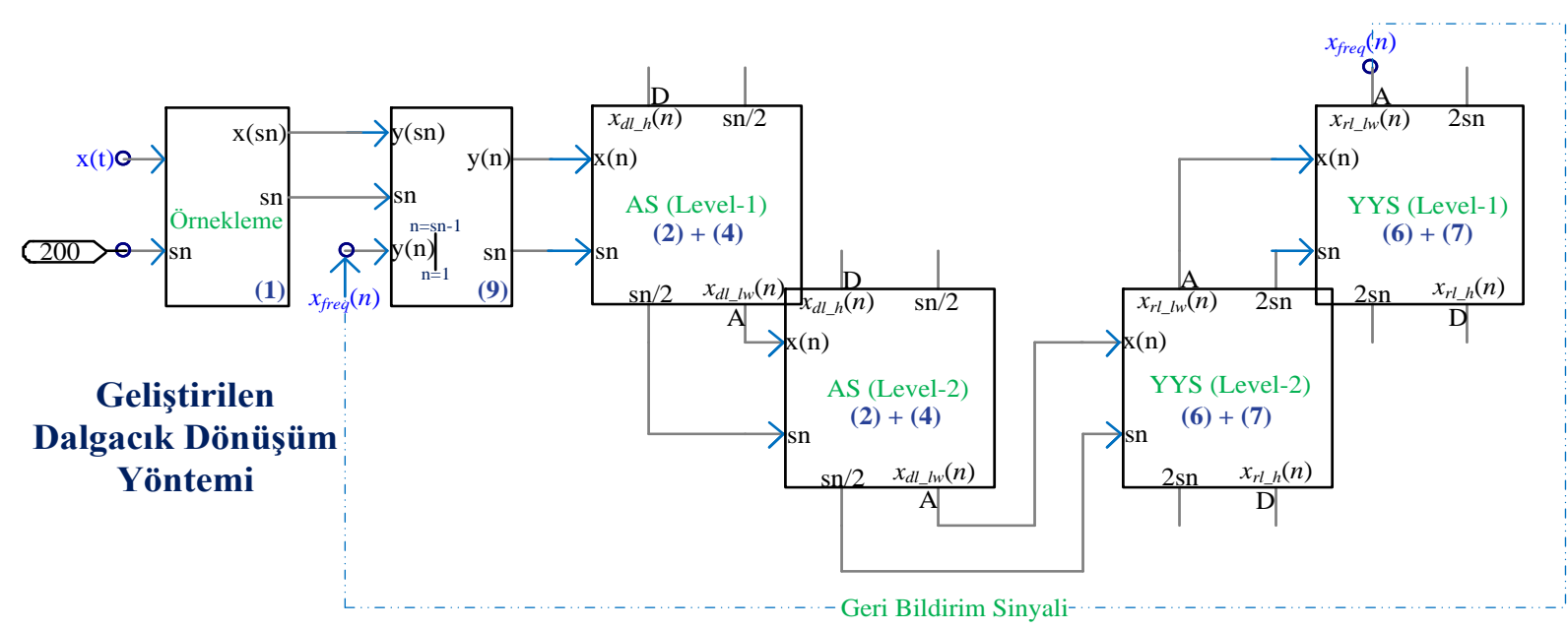

Şekil 3. Geliştirilen Dalgacık Dönüşümü yönteminin detaylı gösterimi (The detailed view of the proposed Wavelet Transform Method) 
Tablo 1. D-STATKOM devre dışı olduğu durumdaki güç sisteminin gerilim ve güç değerleri (The voltage and power ratings of power system without D-STATCOM)

\begin{tabular}{|c|c|c|c|c|c|c|c|c|c|c|c|c|c|c|c|c|c|}
\hline $\begin{array}{l}\text { Örnek } \\
\text { Çalışma }\end{array}$ & Va & phsa & $\mathrm{Vb}$ & phsb & $\mathrm{Vc}$ & phsc & Vab & Vbc & Vac & Pline & Qline & PDC & QDC & PAC & QAC & Plost & Qlost \\
\hline & $\mathrm{kV}$ & $\left({ }^{\circ}\right)$ & $\mathrm{kV}$ & $\left({ }^{\circ}\right)$ & $\mathrm{kV}$ & $\left({ }^{\circ}\right)$ & $\mathrm{kV}$ & $\mathrm{kV}$ & $\mathrm{kV}$ & $\mathrm{kW}$ & kVAr & $\mathrm{kW}$ & $\mathrm{kVAr}$ & $\mathrm{kW}$ & kVAr & $\mathrm{kW}$ & kVAr \\
\hline 1 & 0,22 & 0 & 0,22 & -120 & 0,22 & -240 & 0,38 & 0,38 & 0,38 & 6,14 & 3,36 & 5,80 & 2,37 & 0,00 & 0,00 & 0,34 & 0,99 \\
\hline 2 & 0,2 & 0 & 0,21 & -120 & 0,22 & -240 & 0,38 & 0,38 & 0,38 & 7,03 & 3,86 & 5,62 & 2,28 & 0,96 & 0,26 & 0,45 & 1,32 \\
\hline 3 & 0,18 & -2 & 0,24 & -121 & 0,23 & -238 & 0,36 & 0,40 & 0,36 & 7,13 & 3,86 & 5,61 & 2,29 & 1,06 & 0,25 & 0,46 & 1,32 \\
\hline
\end{tabular}

Tablo 2. Birinci örnek çalışmada D-STATKOM devre dışı ve devredeyken hattın güç değerleri (Power parameters of line when D-STATKOM is online and offline for the first-case)

\begin{tabular}{lllllllll}
\hline D-STATKOM & $\begin{array}{l}\text { Pline } \\
\mathrm{kW}\end{array}$ & $\begin{array}{l}\text { Qline } \\
\mathrm{kVAr}\end{array}$ & $\begin{array}{l}\text { PDC } \\
\mathrm{kW}\end{array}$ & $\begin{array}{l}\text { QDC } \\
\mathrm{kVAr}\end{array}$ & $\begin{array}{l}\text { PAC } \\
\mathrm{kW}\end{array}$ & $\begin{array}{l}\text { QAC } \\
\mathrm{kVAr}\end{array}$ & $\begin{array}{l}\text { Plost } \\
\mathrm{kW}\end{array}$ & $\begin{array}{l}\text { Qlost } \\
\mathrm{kVAr}\end{array}$ \\
\hline Devre Dış1 & 6,140 & 3,360 & 5,800 & 2,370 & 0,000 & 0,000 & 0,340 & 0,990 \\
Devrede & 7,595 & 0,149 & 6,834 & 2,282 & 0,000 & 0,000 & 0,761 & $-2,133$ \\
\hline \multicolumn{2}{l}{ Pline = PDC + PAC + Plost } & \multicolumn{7}{c}{ Qline = QDC + QAC + Qlost }
\end{tabular}

Tablo 1'de dağıtım hattının toplam aktif-reaktif güç değerleri (Pline - Qline), doğrultucunun aktif-reaktif güç değerleri $(P D C-Q D C)$, dengesiz yük olarak sisteme dâhil edilen yükün aktif-reaktif güç değerleri $(P A C-Q A C)$ ve dağıtım hattında kaybolan aktif-reaktif güç değerleri (PlostQlost) verilmiştir. Bu değerler, örnek çalışmalarda DSATKOM devreye alındıktan sonraki değerleriyle karşılaştırılacaktır.

5.1. Örnek Çalışma-1: Dengeli Gerilim Kaynă̆ + Doğrultucu (Case Study-1: Balanced Voltage Source + Rectifier)

Akım ve gerilimde bulunan harmonik etkilerin azaltılmasında genellikle AGF kullanılmaktadır. Önerilen DD metotla, D-STATKOM bu görevi yerine getirebilmektedir. $\mathrm{Bu}$ durum çalışmasında güç devresinde doğrultucuyu devreye alıp, bir harmonik kaynağı olarak çalıştırılmıştır. Doğrultucuda DA yük olarak " $30 \Omega+8 \mathrm{mH}^{\circ}$ değerinde direnç ve indüktans kullanılmıştır. Benzetim süresi 1,0 s (saniye) olup, ilk 0,50s'de D-STATKOM devre dışı bırakılıp, 0,50 s'den sonra devreye alınarak sistem dinamikleri (faz-nötr ve faz-faz gerilimleri, hat ve yük akımları, aktif-reaktif güç değerleri) grafiksel olarak irdelenmiştir. Benzetim çalışmasında 0,0s-0,50s arasında DSTATKOM devre dışı bırakılmış, 0,50s-1,0s saniyeleri arasında devreye alınarak performansı izlenmiş olup, sistemin güç değerleri karşılaştırmalı olarak Tablo 2'de sunulmuştur. Şekil 4-A'da sistemin güç değeri gösterilmiştir. 0,50s-0,60s saniyeleri arasında evirgeçteki kapasitenin gerilim indüklemesi için hattan aktif enerji çekilmektedir. Aktif enerjinin çekilmesi hat akımın artmasına neden olacağından reaktif enerjide aynı oranda artacaktır. Bu sebepten 0,10s süresince Pline - Qline anlık olarak 2 katı kadar yükselmiştir. 0,60s-1,0s saniyeleri arasında hattın aktif enerjisi, reaktif enerji ve harmonik kompanzasyonunda gerekli enerjiyi sağlamak için artarken; reaktif enerji tamamen sıfir düzeylerine çekilmiştir. 732
PSCAD/EMTDC'nin kütüphanesinde bulunan FFT modülü kullanılarak hat akımında \%14,50'lik Toplam Talep Bozunum (TTB) ve hat gerilimlerinde benzer seviyede Toplam Harmonik Bozunum (THB) ölçülmüş olup, bu harmonik oran sistemin faz gerilimlerine de yansımaktadır. $\mathrm{Bu}$ durum Şekil 4-B'de 0,0s-0,50s arasında kolaylıkla görülebilmektedir. 0,50s'de devreye giren D-STATKOM sistemin güç değerlerinde 5 periyot süre boyunca geçici bir yükselmeye sebebiyet vermiş olsa da, bu durum akımgerilim dinamiklerinde sadece 1 periyot sürmüştür. $0,52 \mathrm{~s}-$ 1,0s saniyeleri arasında hem akımda hem de gerilimdeki harmonikler kompanze edilmiş olup, THB seviyesi \%3,05 ve TTB seviyesi \%2,83 kadar düşürülmüştür.

\section{2. Örnek Çalışma-2: Dengeli Gerilim Kaynă̆ + Dengesiz Yük + Doğrultucu \\ (Case Study-2: Balanced Voltage Source + Unbalanced Load + Rectifier)}

Sanayide kullanılan yükler, elektrik şebekesine dengeli olarak dağıtılmaya çalışılmaktadır. Kaynak ve baskı makinaları gibi anlık olarak devreye girip çıkan yükler sistemde dengesiz faz akımı çekilmesine neden olmaktadır. Bu örnek çalışmada sisteme 0,30s'de BRK1 kapalı duruma getirilip dengesiz yük ve 0,50 s'den sonra da BRK2 kapalı duruma getirilip D-STATKOM devreye alınmış olup, sistem dinamiklerinin değişimi Şekil 5'te sunulmuştur. Ayrıca, sistemin güç değerleri karşılaştırmalı olarak Tablo 3 'de verilmiştir. 0,30 s'den sonraya devreye alınan dengesiz yük, aktif ve reaktif enerjide artmaya ve faz akımlarında dengesizliğe neden olmuştur. 0,50s'de D-STATKOM devreye alınmış olup, $0,52 \mathrm{~s}$ 'de akım harmoniğini ve 0,60 s'den sonra sistemin reaktif enerjisini kompanze etmeye başlamıştır. FFT modülü kullanılarak, TTB değeri \%12,30 ve THB değeri \%14,0 olarak kompanzasyon öncesi ölçülmüş olup, kompanzasyon sonrası TTB seviyesi \%5,29 ve THB seviyesi \%4,07 kadar düşürülmüştür. 

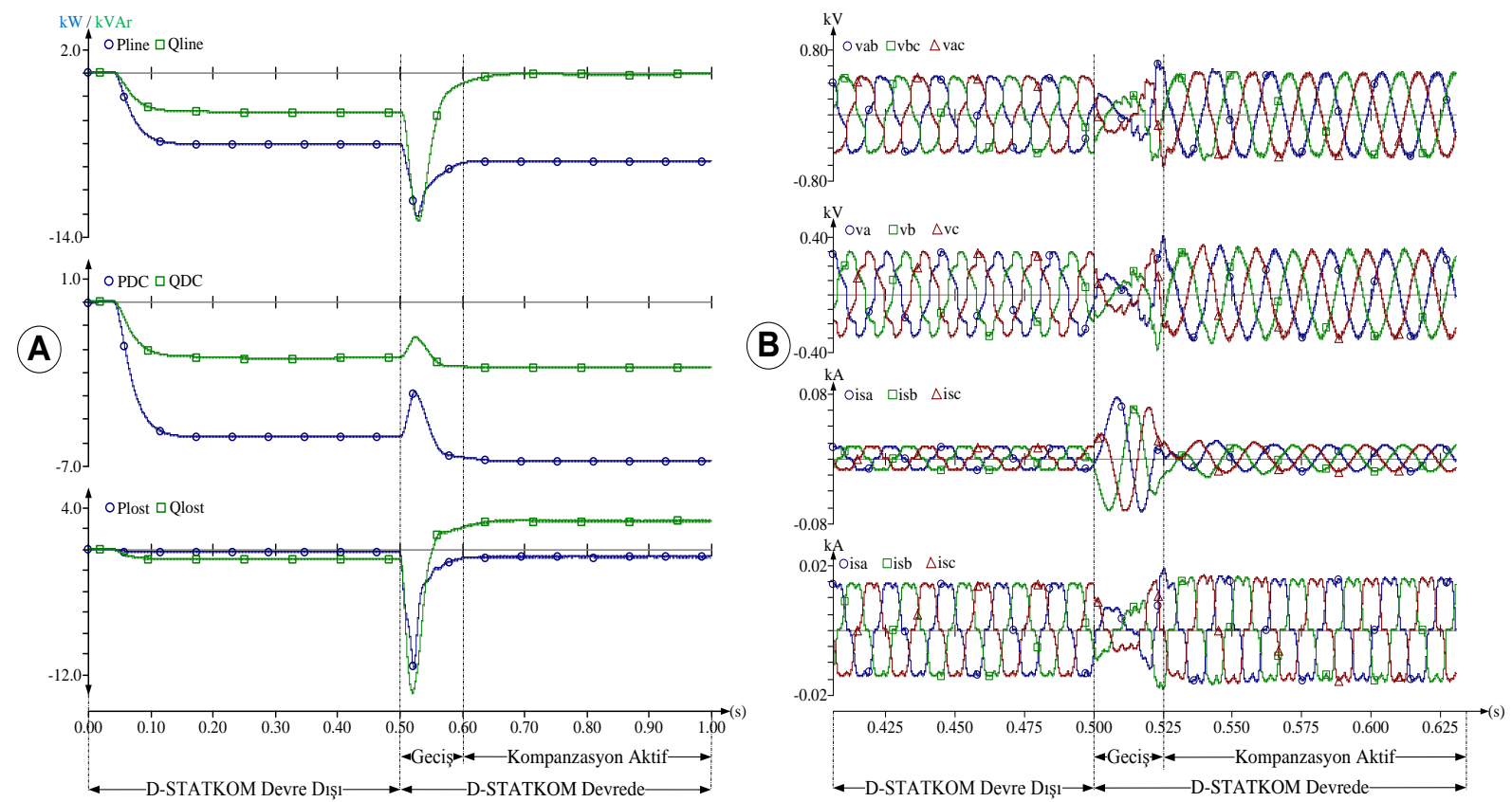

Şekil 4. Birinci örnek çalışmasının benzetim sonuçları (The simulation results of first-case)
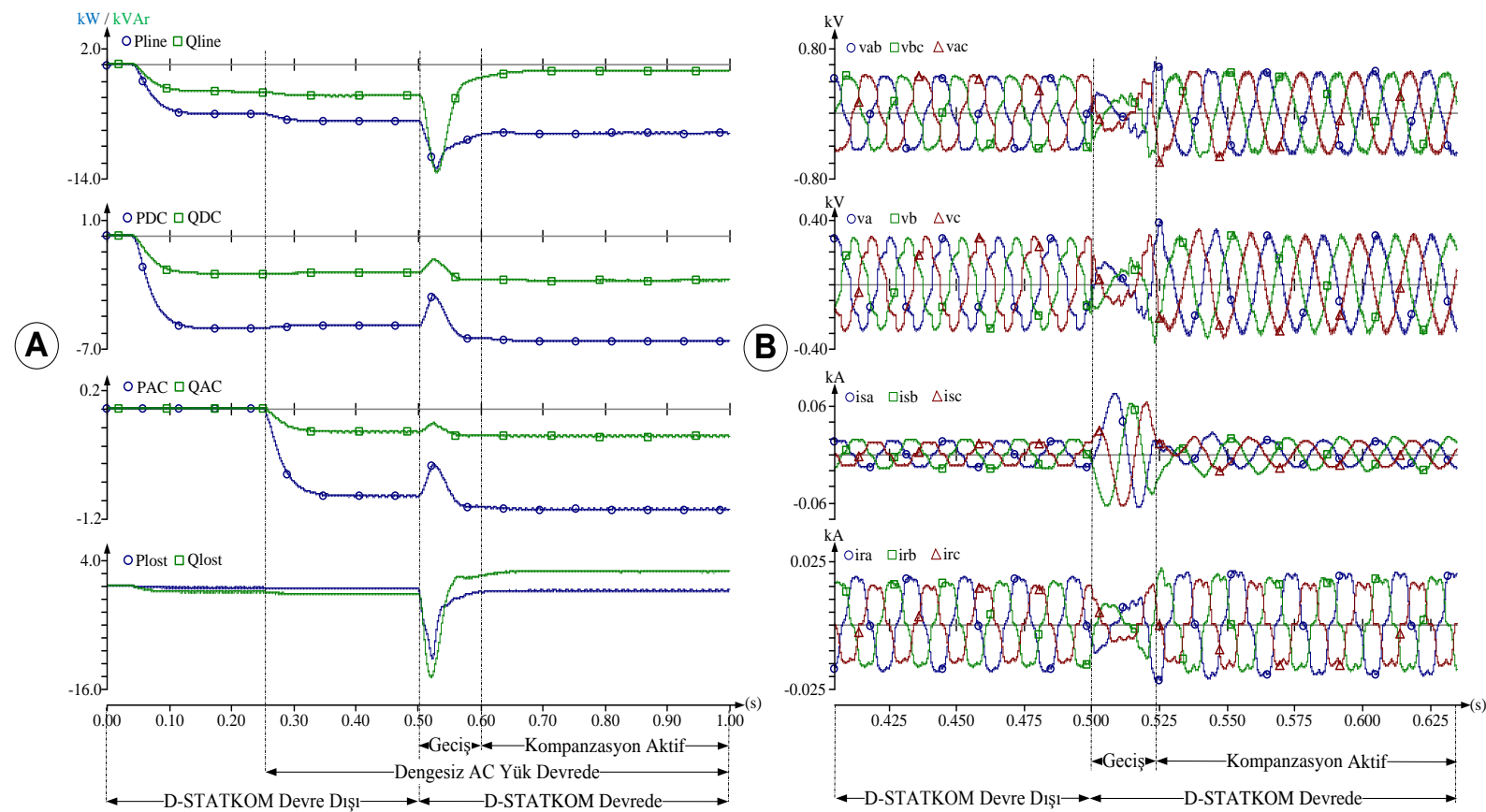

Şekil 5. İkinci örnek çalışmasının benzetim sonuçları (The simulation results of second-case)

Tablo 3. İkinci örnek çalışmada D-STATKOM devre dışı ve devredeyken hattın güç değerleri (Power parameters of line when D-STATKOM is online and offline for the second-case)

\begin{tabular}{lllllllll}
\hline \multirow{2}{*}{ D-STATKOM } & $\begin{array}{l}\text { Pline } \\
\mathrm{kW}\end{array}$ & $\begin{array}{l}\text { Qline } \\
\mathrm{kVAr}\end{array}$ & $\begin{array}{l}\mathrm{PDC} \\
\mathrm{kW}\end{array}$ & $\begin{array}{l}\mathrm{QDC} \\
\mathrm{kVAr}\end{array}$ & $\begin{array}{l}\mathrm{PAC} \\
\mathrm{kW}\end{array}$ & $\begin{array}{l}\mathrm{QAC} \\
\mathrm{kVAr}\end{array}$ & $\begin{array}{l}\text { Plost } \\
\mathrm{kW}\end{array}$ & $\begin{array}{l}\text { Qlost } \\
\mathrm{kVAr}\end{array}$ \\
\hline \multirow{2}{*}{ Devre Dış1 } & 7,030 & 3,860 & 5,620 & 2,280 & 0,958 & 0,262 & 0,452 & 1,318 \\
Devrede & 8,461 & 0,560 & 6,549 & 2,775 & 1,105 & 0,309 & 0,807 & $-2,524$ \\
\hline
\end{tabular}

Pline $=$ PDC + PAC + Plost

Qline $=$ QDC + QAC + Qlost 
Tablo 4. Üçüncü örnek çalışmada D-STATKOM devre dışı ve devredeyken hattın güç değerleri (Power parameters of line when D-STATKOM is online and offline for the third-case)

\begin{tabular}{lllllllll}
\hline D-STATKOM & $\begin{array}{l}\text { Pline } \\
\mathrm{kW}\end{array}$ & $\begin{array}{l}\text { Qline } \\
\mathrm{kVAr}\end{array}$ & $\begin{array}{l}\mathrm{PDC} \\
\mathrm{kW}\end{array}$ & $\begin{array}{l}\text { QDC } \\
\mathrm{kVAr}\end{array}$ & $\begin{array}{l}\text { PAC } \\
\mathrm{kW}\end{array}$ & $\begin{array}{l}\mathrm{QAC} \\
\mathrm{kVAr}\end{array}$ & $\begin{array}{l}\text { Plost } \\
\mathrm{kW}\end{array}$ & $\begin{array}{l}\text { Qlost } \\
\mathrm{kVAr}\end{array}$ \\
\hline Devre Diş1 & 7,129 & 3,856 & 5,612 & 2,288 & 1,059 & 0,248 & 0,458 & 1,320 \\
Devrede & 8,531 & 0,472 & 6,518 & 2,752 & 1,198 & 0,277 & 0,815 & $-2,557$ \\
\hline
\end{tabular}
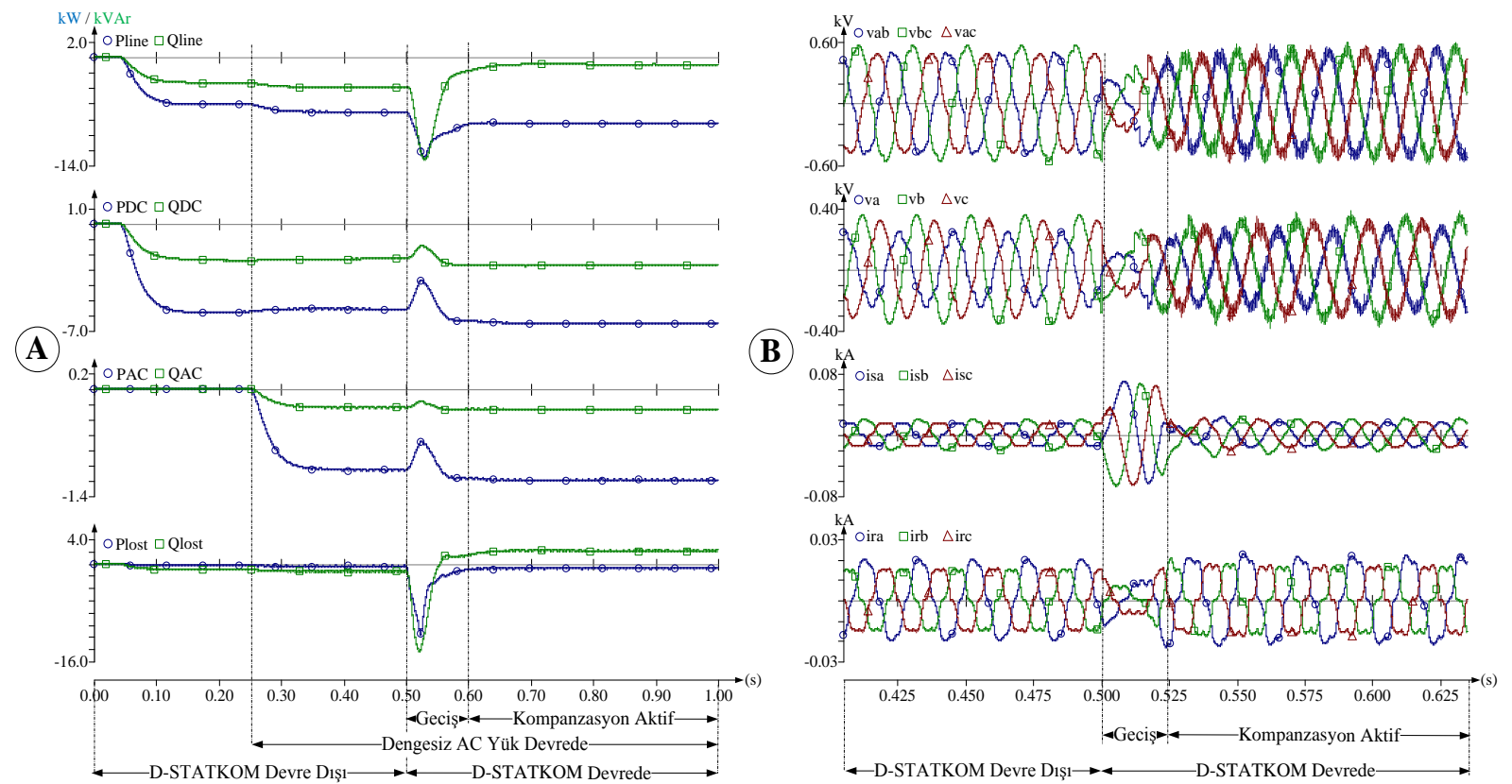

Şekil 6. Üçüncü örnek çalışmasının benzetim sonuçları (The simulation results of third-case)

\section{3. Örnek Çalışma-3: Dengesiz Gerilim Kaynă̆ +} Dengesiz Yük + Doğrultucu

(Case Study-3: Unbalanced Voltage Source + Unbalanced Load + Rectifier)

Elektrik üreticilerinden kaynaklanan hatalardan dolayı dağıtım hatlarında dengesiz gerilimler oluşmaktadır. $\mathrm{Bu}$ hatalardan dolayı üç-faz ile beslenen yüklerde bozulmalara ve dengesiz yüklenmelere neden olmaktadır. $\mathrm{Bu}$ örnek çalışmada kaynak gerilim değerleri Tablo 1'de verildiği şekilde değiștirilmiş olup, D-STATKOM' ile hatlar arası gerilimlerinin normal değerlerinde tutulmaya çalışılmıştır. Benzetim çalışmasında sisteme 0,30s'de BRK1 kapalı duruma getirilip dengesiz yük ve 0,50s'den sonra da BRK2 kapalı duruma getirilip D-STATKOM devreye alınmış olup, sistem dinamiklerinin değişimi Şekil 6'da sunulmuştur. Ayrıca, sistemin güç değerleri karşılaştırmalı olarak Tablo 4'de verilmiştir. D-STATKOM 0,50s'den sonra devreye alınmış olup, 0,60s'den sonra harmonik ve reaktif enerjiyi etkili bir şekilde kompanze etmeye başlamıştır. Ayrıca, 0,50s'e öncesi hatlar arası gerilim dengesizliği (Tablo 1'de belirtilen hat ve faz gerilimleri değerleri) reaktif güç kompanzasyonu ile giderilmiş ve faz-nötr gerilim dengesizlikleri azaltılmaya çalıșılmıștır. FFT modülü kullanılarak, TTB değeri $\% 18,40$ ve THB değeri $\% 12,76$ olarak kompanzasyon öncesi ölçülmüş olup, kompanzasyon sonras1 TTB seviyesi $\% 5,23$ ve THB seviyesi \%4,37 kadar düşürülmüştür.

\section{SONUÇLAR (CONCLUSIONS)}

D-STATKOM hem dağıtım hem de iletim sistemlerinde kullanılan evirgeç tabanlı şebekeye paralel bağlı aygıt olup güç devresi temel olarak DA kapasitör, evirgeç, çıkış filtresi ve gerekli olması durumunda şebekeye bağlantı sağlayan transformatörden oluşmaktadır. D-STATKOM sistemleri, $\pm \mathrm{VAr}$ aralığında, şebeke frekansında $(50 \mathrm{~Hz})$ sürekli, hem indüktif hem de kapasitif reaktif güç üretebilmektedir. Bu yeteneklerinden dolayı, dağıtım ve iletim sistemlerinin GK problemlerinin çözümünde doğrudan kullanılabilecek geleceğin teknolojisi olarak görülmektedir. Bu çalışmada ilk defa, kontrol yöntemi Genel Dalgacık Dönüşümünden geliștirilen bir D-STATKOM önerilmiștir. Önerilen DD algoritması, geri-bildirim yöntemi ile giriş sinyalin temel harmoniğini en kısa sürede ulaşılabilirliğini arttırmış olup, tepki süresi daha önceki sürümlerine göre daha hızlandırılmıştır. Böylelikle DD’nin elektriksel güç uygulanabilirliği arttırılmıştır. Ayrıca, bu çalıșma ile birlikte 
genel dalgacık algoritması literatürde ilk defa matematiksel olarak formüle edilmiştir. Durum çalışmalarında önerilen yöntem ile hem akım/gerilim harmonikleri hem de reaktif enerji başarılı bir şekilde kompanze edilmiş olup, yük karakteristiklerinden dolayı ortaya çıkan olumsuz sistem koşulları giderilmiştir. Üç adet durum çalışmasında aynı güç devresi kullanılmış ancak kesiciler kullanılarak farklı durum çalışmaları oluşturulmuştur. Devrede kullanılan dağıtım hattı modülü üzerinde bir gerilim düşümü gerçekleşmektedir. $\mathrm{Bu}$ durum doğrultucu ile birlikte dengesiz yük modülü üzerinde $0,22 \mathrm{kV}$ 'dan düşük gerilim düşümüne sebep olmaktadır. D-STATKOM sisteme reaktif enerji vererek hem kompanzasyon hem de gerilim düzenlemesini aynı anda gerçekleştirmektedir. Bu durum örnek çalışmalarda $0,0 \mathrm{~s}-0,50 \mathrm{~s}$ arasında gerilim düzeyi ile 0,50s-1,0s arasındaki gerilim düzeylerinin karşılaştırılmasında daha kolay fark edilecektir. Birinci örnek çalışmasında dengeli gerilim kaynağına sahip bir sistem oluşturulmuş ve harmonik kaynağı olarak 6-darbeli doğrultucu kullanılmıştır. STATKOM devreye girdikten sonra hat akımındaki \%14,50'lik THB \%3,05'e kadar düşürülmüştür. İkinci örnek çalışmasında sisteme dengesiz yük devreye alınmış ve böylece yük üzerinde dengesiz bir gerilim oluşturulmuştur. Durum çalışması sonuçlarında TTB değeri \%12,30Den \%5,29'a ve THB değeri \%14,0'dan \%4,07'e kadar düşürülmüş ve kompanzasyon öncesi yükte oluşan gerilim bozukluğu, D-STATKOM devreye alındıktan sonra giderilmiştir. Şebeke tarafından kaynaklanan dengesiz gerilim çukuru-tepeleri ve faz atlamaları üçüncü örnek çalışmada sistem gerilim kaynağının faz gerilimleri değiştirerek gerçeklemeye çalışılmıştır. Bu durumda D-STATKOM hem hatlar arası dengesiz gerilim dalgalanmasını düzenlemiş hem de gerilim düzenlemesi yaparak şebeke tarafından kaynaklanan dengesiz sistem koşullarının giderilmesi yerine getirilmiştir. TTB değeri \%18,40'den \%5,23'e ve THB değeri $\% 12,76$ 'den \%4,37'e kadar düşürülmüştür.

\section{TEŞEKKÜRLER (ACKNOWLEDGEMENT)}

Bu çalışma TÜBİTAK Kurumu tarafindan 5-2014 / 5-2015 tarihli ve 144E015 numaralı proje adı altında desteklenmiş olup, kuruma teşekkürlerimizi sunarız.

\section{KAYNAKLAR (REFERENCES)}

1. Sepulveda C. A., Munoz J. A., Espinoza J. R., Figueroa M. E., All-on-chip DQ-frame based D-STATCOM control implementation in a low-cost FPGA, IEEE Transactions on Industrial Electronics, 60 (2), 659-669, 2013.

2. Zaveri T., Bhalja B., Zaveri N., Load compensation using DSTATCOM in three-phase, three-wire distribution system under various source voltage and delta connected load conditions, International Journal of Electrical Power \& Energy Systems, 41 (1), 34-43, 2012.

3. Karmiris G., Tsengenes G., Adamidis G., A multifunction control scheme for current harmonic elimination and voltage sag mitigation using a three phase three level flying capacitor inverter, Simulation Modelling Practice and Theory, 24, 15-34, 2012.

4. Solanki J., Frohleke N., Bocker J., Wallmeier P., Analysis, design and control of $1 \mathrm{MW}$, high power factor and high current rectifier system, IEEE Congress and Exposition on Energy Conversion, 1725-1732, 2012.

5. Arya R.S., Singh B., Power quality improvement under nonideal AC mains in distribution system, Electric Power Systems Research, 106, 86-94, 2014.

6. Arya S.R., Singh B., Performance of DSTATCOM Using Leaky LMS Control Algorithm, IEEE Journal of Emerging and Selected Topics in Power Electronics, 1 (2), 104-113, 2013.

7. Singh B., Arya S.R., Implementation of single-phase enhanced phase-locked loop-based control algorithm for three-phase DSTATCOM, IEEE Transactions on Power Delivery, 28 (3), 1516 -1524, 2013.

8. Singh B., Arya S.R,. Back-propagation control algorithm for power quality improvement using DSTATCOM, IEEE Transactions on Industrial Electronics, 61 (3), 1204-1212, 2014.

9. Singh B., Arya S. R, Composite observer-based control algorithm for distribution static compensator in fourwire supply system, IET Power Electronics, 6 (2), 251260, 2013.

10. Arya S.R., Singh B., Implementation of distribution static compensator for power quality enhancement using learning vector quantisation, IET Generation, Transmission \& Distribution, 7 (11), 1244-1252, 2013.

11. Singh B., Arya S.R., Chandra A., Al-Haddad K., Implementation of adaptive filter based control algorithm for Distribution Static Compensator, IEEE Annual Meeting on Industry Applications Society, 711, 2012.

12. Mishra M.K., Ghosh A., Joshi A., Operation of a DSTATCOM in voltage control mode, IEEE Transactions on Power Delivery, 18 (1), 258-264, 2003.

13. Arya S.R, Singh B., Chandra A., Al-Haddad K., Control of shunt custom power device based on AntiHebbian learning algorithm, 38th IEEE Annual Conference on Industrial Electronics Society, 12461251, 2012.

14. Arya S.R., Singh B., CTF control algorithm of DSTATCOM for Power factor correction and zero voltage regulation, IEEE Third International Conference on Sustainable Energy Technologies, 157162, 2012.

15. Arya S.R., Singh B., Chandra A., AI-Haddad K., Control of DSTATCOM using adjustable step least mean square control algorithm, 5th IEEE Fifth Power India Conference, 1-6, 2012.

16. Singh B., Solanki J., A comparison of control algorithms for DSTATCOM, IEEE Transactions on Industrial Electronics, 56 (7), 2738-2745, 2009. 
17. Elnady A., Salama M.M., A, Unified approach for mitigating voltage sag and voltage flicker using the DSTATCOM, IEEE Transactions on Power Delivery, 20 (2), 992-1000, 2005.

18. Shukla A., Joshi A., State feedback control of multilevel inverters for DSTATCOM Applications, IEEE Transactions on Power Delivery, 22 (4), 24092418, 2007.

19. Singh B., Jayaprakash P., Kothari D.P., New control approach for capacitor supported DSTATCOM in three-phase four wire distribution system under nonideal supply voltage conditions based on synchronous reference frame theory, International Journal of Electrical Power \& Energy Systems, 33 (5), 11091117, 2011.

20. Aggarwal M., Gupta S.K., Singh M., Analysis and control of grid connected wind energy system with DSTATCOM, IEEE Fifth Power India Conference, 16, 2012.

21. George V., Mishra M.K., Design and analysis of userdefined constant switching frequency current-controlbased four-leg DSTATCOM, IEEE Transactions on Power Electronics, 24 (9), 2148-2158, 2009.

22. Srikanthan S., Mishra M.K., Rao R.K.V., Improved hysteresis current control of three-level inverter for distribution static compensator application, IET Power Electronics, 2 (5), 517-526, 2009.

23. Shukla A., Ghosh A., Joshi A., Hysteresis current control operation of flying capacitor multilevel inverter and its application in shunt compensation of distribution systems, IEEE Transactions on Power Delivery, 22 (1), 396-405, 2007.

24. Reddy J.G.P., Reddy K.R., Power quality improvement using Neural Network controller based cascaded $\mathrm{H}$ Bridge multilevel inverter type D-STATCOM, International Conference on Computer Communication and Informatics, 1-6, 2012.

25. Bhattacharjee C., Roy A.K., Roy B.K., Improvement of available load voltage for a constant speed WECS coupled with fuzzy-controlled DSTATCOM, 15th International Conference on Harmonics and Quality of Power, 637-641, 2012.

26. Nijhawan P., Bhatia R.S., Jain D.K., Role of DSTATCOM in a power system network with induction furnace load, IEEE Fifth Power India Conference, 1-5, 2012.

27. Singh B., Arya S.R., Admittance based control algorithm for DSTATCOM in three phase four wire system, 2nd International Conference on Power, Control and Embedded Systems, 1-8, 2012.

28. Bukata B.B., Li Y., A novel model-free prediction of power quality problems via DSTATCOM, 18th International Conference on Automation and Computing, 1-6, 2012.
29. Mishra M K., Karthikeyan K., A fast-acting dc-link voltage controller for three-phase DSTATCOM to compensate AC and DC loads, IEEE Transactions on Power Delivery, 24 (4), 2291-2299, 2009.

30. Gupta R., Ghosh A., Frequency-domain characterization of sliding mode control of an inverter used in DSTATCOM application, IEEE Transactions on Circuits and Systems I: Regular Papers, 53 (3), 662676, 2006.

31. Wang H., Meng X., Zhao L., Zhao T., Controlling study of D-STATCOM based on Hybrid Genetic Optimization PID algorithm, 24th Chinese Conference on Control and Decision, 2762-2766, 2012.

32. Singh B., Arya S.R., Adaptive theory-based improved linear sinusoidal tracer control algorithm for DSTATCOM, IEEE Transactions on Power Electronics, 28 (8), 3768-3778, 2013.

33. Mitra P., Venayagamoorthy G.K., An adaptive control strategy for DSTATCOM applications in an electric ship power system, IEEE Transactions on Power Electronics, 25 (1), 95-104, 2010.

34. Sünter Z., Altun H., Sünter S., A New Approach for Harmonic Elimination in Single-Pulse Modulated Single-Phase Inverter Drive System, Journal of the Faculty of Engineering and Architecture of Gazi University, 30 (2), 237-247, 2015.

35. Kumar C., Mishra M.K., A modified D-STATCOM topology with reduced VSI rating, DC link voltage, and filter size, 2013 International Conference on Clean Electrical Power (ICCEP), 325-331, 2013.

36. Miller T.J.E, Hanselman D., Janhs T.M., Mcdonald J., Power electronic control in electrical systems, Newnes Power Engineering Series, 2002.

37. Pittorino L.A., Hom A., Enslin J.H.R., Power theory evaluation for the control of an active power filter, IEEE AFRICON 4th, 2 (4), 676-681, 1996.

38. Lee S.J., Kang J.K., Sul S.K., A new phase detecting method for power conversion systems considering distorted conditions in power system, Thirty-Fourth IAS Annual Meeting Industry Applications Conference, 4 (34), 2167-2172, 1999.

39. Teke A., Meral E.M., Saribulut L., Tümay M., Implementation of fuzzy logic controller using FORTRAN language in PSCAD/EMTDC, International journal of electrical engineering education, 48 (4), 372-390, 2011.

40. Üstündağ M., Avcı E., Gökbulut M., Ata F., Denoising of Weak Radar Signals Using Wavelet Packet Transform and Genetic Algorithm, Journal of the Faculty of Engineering and Architecture of Gazi University, 29 (2), 375-383, 2014. 